\title{
Bursting with potential
}

With a decade under its belt, the study of fast radio bursts looks set for a revelatory next few years as some of the key questions driving the field forward will be answered. In this issue, we look back at how things started and look ahead to what is to come.

T his issue of Nature Astronomy, and particularly its Focus on fast radio bursts (FRBs), has been in the planning for several months. FRBs are very energetic and so far sporadic radio signals that are of unknown - but probably cosmological - origin. This Focus is extremely timely, since almost as many new FRB sources have been reported in the $\sim 12$ weeks it took to write and edit the component articles than in the preceding 12 years. Such is the rapid development of this field. Much of this deluge of FRB detections has been due to early results from new facilities, most notably the Australian Square Kilometre Array Pathfinder (ASKAP), which features on our cover, and the Canadian Hydrogen Intensity Mapping Experiment (CHIME), which just broke new ground by recording the lowest-frequency FRB. ASKAP is the subject of our Mission Control this month. And we heard about CHIME back in Issue 2.

ASKAP or CHIME might be to FRBs what BeppoSAX was to gamma-ray bursts (GRBs). To fully understand what that means, read the Comment by Shri Kulkarni. Using the wisdom of ages, Kulkarni draws insightful parallels between the mature study of GRBs (45 years old this year) and the study of FRBs, in its adolescence. There are valuable lessons to be learnt for the astronomer who has time for hindsight.

Duncan Lorimer is such an astronomer. Just over ten years ago, Lorimer and his student David Narkovic came across an odd radio signal in some archival pulsar data. Looking for unusual radio pulses had suddenly become all the rage in those days - Maura McLaughlin had discovered the pulsar-like RRATs (rotating radio transients) a few years earlier through a similar process. But it took the combined efforts of Lorimer, McLaughlin, Narkovic and colleague Matthew Bailes to truly understand that the signal was astrophysical, and that it was something different to what had been seen before. That initial FRB discovery paper, published in 2007, made some bold statements about the origin of that 30-jansky, 5-millisecond-long burst: it came from a cosmological distance, but within a gigaparsec; it did not repeat, indicating a catastrophic origin; hundreds of these bursts could be hitting the Earth every day; and these bursts could be used as probes of the Universe. These statements are still valid today. Lorimer recounts this discovery, and the first decade of FRB studies that it inspired, in a Perspective at the heart of this Focus issue.

After steady, but slow, progress, a major discovery was made in the field just over two years ago: an FRB source that sends repeat bursts sporadically... the now-famous FRB 121102. To date it remains the only FRB to exhibit this recurrent behaviour. What does this mean for the catastrophic model of FRB generation? It is still not clear whether FRB 121102 is a representative of a distinct class of FRB sources, or whether it might be from the same singular population, but at a different evolutionary stage. Manisha Caleb, Laura Spitler and Ben Stappers dig further into the details in a Comment in this issue.

As we begin the second decade of FRB science, and in particular after a fecund October, when 20 FRBs detected with ASKAP were reported by Ryan Shannon et al. in Nature (we have a Research Highlight to get you up to speed), studies can now start considering statistics. Evan Keane takes on the challenge of looking to the intermediate and far future of the field in a Review Article. Now we have $\sim 60$ FRBs to study, and in the coming years Keane expects this to grow to thousands. With these numbers, some of the most pressing unanswered questions will surely be answered: what is the nature of FRBs? (Ue-Li Pen also looks at this question in detail in his Comment.) Are FRBs exclusively a radio phenomenon, or are there counterparts at other wavelengths or using other messengers? (Sarah Burke-
Spolaor gives her in-depth attention to this question in her Comment.) How can we use FRBs to probe the cosmos? (Jean-Pierre Macquart takes us back almost to the dawn of time in his Comment.)

The next step to advance many of these aspects will be localization. The source of just one FRB (121102) has been localized, following a concerted effort. Just that one localization now means that we know considerably more than we did two years ago about where FRBs are generated. In this case, the burst left the star-forming outskirts of an optically faint, irregular, low-metallicity dwarf galaxy at a redshift of $z \approx 0.2$. To all those descriptors we can add more: analysis of 16 bursts indicates that FRB 121102 came from "an extreme and dynamic magneto-ionic environment, and the short durations of the bursts suggest a neutron star origin." And this is without the 72 bursts recently discovered by machine learning techniques in Green Bank Telescope data (this Research Highlight has the details) in which is encoded key information about the immediate burst environment. We should remember that FRB 121102 is currently an outlier (in several ways): arcsecond localization of a non-repeating FRB will be the next significant advance in the field.

It feels as though the field of fast radio bursts is on the brink of self-realization. After the best part of a decade of doubt, FRBs are now officially a 'thing. Keane has stories from the early 2010s about being rejected for jobs because studying FRBs was too 'niche' a field. Shortly, as several of the authors that contributed to this Focus issue can attribute, we will be in prime place to exploit FRBs as tools to explore the contents of the Universe way back until the epoch of reionization. Not so niche now.

Published online: 31 October 2018 https://doi.org/10.1038/s41550-018-0640-8 